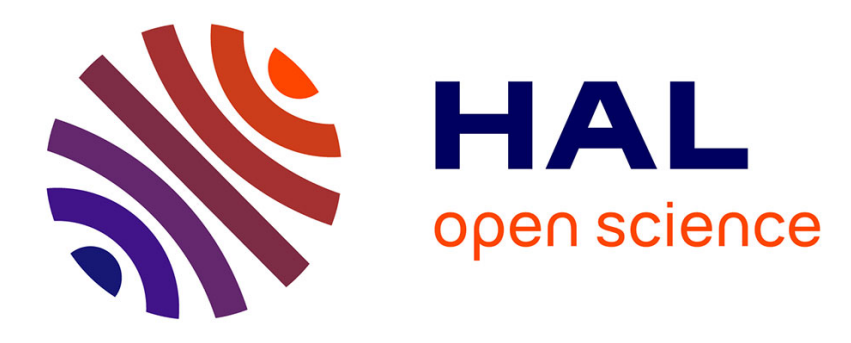

\title{
La dégradation de la structure des sols forestiers Philippe Duchaufour
}

\section{To cite this version:}

Philippe Duchaufour. La dégradation de la structure des sols forestiers. Revue forestière française, 1953, 10, pp.657-665. 10.4267/2042/26933 . hal-03384211

\section{HAL Id: hal-03384211 \\ https://hal.science/hal-03384211}

Submitted on 18 Oct 2021

HAL is a multi-disciplinary open access archive for the deposit and dissemination of scientific research documents, whether they are published or not. The documents may come from teaching and research institutions in France or abroad, or from public or private research centers.
L'archive ouverte pluridisciplinaire HAL, est destinée au dépôt et à la diffusion de documents scientifiques de niveau recherche, publiés ou non, émanant des établissements d'enseignement et de recherche français ou étrangers, des laboratoires publics ou privés. 


\section{LA DÉGRADATION DE LA STRUCTURE DES SOLS FORESTIERS}

Les propriétés physiques des sols sont liées à deux notions fondamentales: la texture - composition élémentaire, lorsque tous les agrégats ont été détruits - et la structure - manière dont ces éléments sont groupés en agrégats plus ou moins gros. D’après la texture, on classe les sols èn sols sableux, limoneux, argileux, etc... La structure, au contraire, est en rapport avec l'état des colloïdes du sol qui sont, tantôt à l'état dispersé (structure dispersée ou particulaire), tantôt à l'état floculé: ils jouent alors le rôle de ciments, vis-à-vis des particules plus grossières et sont responsables de la structure en agrégats ou en grumeaux.

Dans les sols forestiers, les agrégats ainsi formés présentent une dimension et une stabilité variables: Dans les sol's bruns à Mull, légèrement acides, les agrégats sont fins (ils mesurent fréquemment moins d'un millimètre). En outre, ils sont souvent peu stables et peuvent être détruits, par exemple, après une hydratation brutale, suivie elle-même d'une dessiccation rapide (action du soleil succédant à une pluie violente); une dessiccation lente, au contraire, provoque ta reconstitution des agrégat's. Dans les rendzines, les sols riches en calcium. les agrégats sont beaucoup plus gros $(2$ à $5 \mathrm{~mm}$ ) et nettement plus stables: ce sont de véritables grumeaux, tantôt arrondis, tantôt au contraire munis d'arêtes anguleuses, et qui résistent à l'action des pluies.

Le type de structure du sol forestier et sa stabilité sont liés à deux facteurs essentiels :

a) La nature et l'abondance des ciments, argile et humus. L'humus joue le rôle le pluts important; suivant qu'il prend naissance en milieu calcaire (humus calcique) ou en milieu acide, il est constitué par des acides humiques naturellement floculés ou, au contraire, ayant tendance à se disperser; l'humus très acide (Mor) est toujours à l'état dispersé.

Certains auteurs allemands (SCheffer et Welte-Springer) ont pu montrer que les divers acides humiques offrent des propriétés différentes, suivant la richesse en calcium du milieu et la nature des débris organiques qui les engendrent.

Ainsi, ils distinguent:

I. - Les acides humiques gris, formés en milieu calcaire, normalement floculés de façon très stable (humus calcique). 
2. - Les acides humiques bruns, formés en milieu peu acide, floculés de façon instable et pouvant se disperser sous l'action des pluies (humus doux. Mull).

3. - Les complexes ligno-humiques, très acides et toujours dispersés (humus brut ou Mor des sols podzoliques).

b) L'activité biologique. Les vers de terre jouent un rôle important, en régénérant constamment une structure grumeleuse assez stable, lorsqu'elle a tendance à se dégrader: toute destruction de structure non reversible, donc durable, est causée par une diminution grave de l'activité biologique et en particulier animale.

Le tableau suivant résume la relation, qui existe entre les 3 types. fondamentaux de structure et les types de sol et d'humus.

\section{StRUCTURE Des PRINCIPAUX SOLS Forestiers (Hor. $A_{1}$ )}

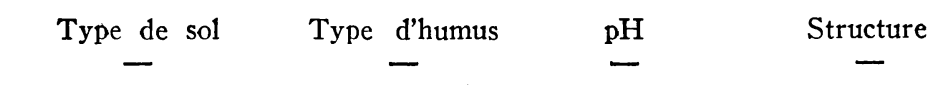

Rendzine et

Rendzine dégradée : humus calcique 6,5 à 7,5 gros $\begin{aligned} & \text { grumeaux stables } \\ & \text { (2 à } 5 \mathrm{~mm} \text { ) }\end{aligned}$

Sol brun ou

Sol lessivé

Mull

$5-6$

petits agrégats

plus ou moins stables

Sol podzolique ou

Sol à Gley ...... Mor sec ou $\quad<5 \quad$ structure particulaire

Mor tourbeux ou dispersée

N. B.: Il s'agit de la structure de l'horizon $A_{1}$, c'est-à-dire d'un horizon mixte, organo-minéral, situé en dessous de la couche d'humus brut $A_{0}$, lorsqu'elle existe.

\section{I. - Rôle De LA STRUCtURE}

La structure joue un rôle essentiel, parce qu'elle maintient les propriétés physiques favorables du sol forestier et aussi parce qu'elle prévient toute évolution pédologique défavorable du profil.

\section{$\mathrm{I}^{\circ}$ La structure maintient des propriétés physiques favorables.}

Elle intervient d'abord dans l'aération et le drainage du sol; alors qu'une structure dégradée est " asphyxiante ", par suite du gonflement des colloïdes en présence d'eau, la structure en agrégats permet au contraire une perméabilité et une aération satisfaisantes. La fraction de l'eau de pluie, qui n'est pas retenue par le sol, circule par gravité dans les vides les plus grossiers qui entourent les grumeaux (leur volume global constitue la " porosité non capillaire " 
du sol). Elle est rapidement remplacée par l'air, au cours du ressuyage.

De plus, la structure en agrégats conditionne également la formation de réserves d'eau profondes, emprisonnées par capillarité clans les pores les plus fins, existant dans la masse même des agrégats (leur volume global constitue la " porosité capillaire »). Cette eau de rétention capillaire se répartit à une profondeur d'autant plus grande que la perméabilité est meilleure et reste ainsi à la disposition des plantes, même si le sol s'assèche en surface, en période chaude.

On voit donc que les mesures concernant la porosité (capillaire et non capillaire) renseignent efficacement sur l'état de la structure du sol : dans un sol forestier en bon état, la porosité non capillaire doit être du même ordre de grandeur que la porosité capillaire; son abaissement marqué trahit une tendance à la dégradation de la structure.

\section{$2^{\circ}$ Une bonne structure prévient toute évolution pédologique nuisible.}

La circulation aisée de l'eau d'infiltration, dans les pores grossiers du sol entourant les agrégats, évite ou réduit au minimum les phénomènes d'entraînement des colloïdes; le lessivage est presque nul dans les sols à structure très stable (rendzines, sols bruns riches en calcium). Dans les sols à humus doux plus acide, une dégradation partielle de la structure se produisant, en saison humide, peut entraîner un certain lessivage des colloïdes: mais ce lessivage est rapiđement compensé par les phénomènes de remontée exercés par l'activité animale, en général intense dans ces sols bien aérés. Sous son influence, la structure se reconstitue, en saison plus favorable.

Ainsi, tant que l'humus ne s'acidifie pas de façon marquée, le lessivage reste limité.

En outre, la structure en agrégats assurant une bonne circulation de l'air, aucun phénomène de réduction de sels de fer ne peut se produire: ceux-ci restent à l'état ferrique. Ces hydrates ferriques liés, sous forme de complexes, à l'argile et à l'humus, au sein même des agrégats, confèrent au sol une couleur brune caractéristique d'une structure favorable. Un sol forestier en bon état est donc, pédologiquement, un sol brun ou, plus généralement, un sol brun lessivé, dans le cas où un lessivage réduit s'est produit.

\section{II. - Causes et conséQuences de la dÉGRAdation DE LA STRUCTURE}

Ainsi, la forêt feuillue en bon état maintient une structure suffisamment stable en surface, grâce à l'humus doux qu'elle produit et à la grande activité biologique qui caractérise son sol. 
Mais certains sols sont en équilibre instable, soit par: suite d'un excès, soit au contraire par suite d'une insuffisance de colloïdes. Sur ces sols, toute cause provoquant une acidification de l'humus et une décroissance de l'activité biologique est le point de départ. d'une dégradation de la structure.

Ces causes peuvent être de deux ordres:

$\mathrm{I}^{\circ}$ Une dénudation fréquente ou prolongée $d u$ sol à la suite de coupes non suivies d'une régénération immédiate; sous l'action des radiations solaires, l'humus se minèralise et disparait; les agrégats, privés de leur ciment, se détruisent et les argiles ont tendance à se disperser. En outre, les espèces acidiphiles, notamment les Bruyères, se multiplient et arrivent parfois à envahir le parterre de la coupe: elles engendrent alors un humus plus acide et plus dispersé que l'humus forestier primitif, ce qui ne peut qu'accélérer le processus de dégradation précédemment amorcé par l'exposition au soleil.

A ce point de vue, nous avons insisté, dans une précédente étude [5], sur la nécessité de réussir le plus rapidement possible les régénérations, de façon à ne pas laisser le sol découvert.

Dans le même ordre d'idées, les coupes de taillis à courte révolution, dénudant le sol à intervalles répétés, exercent une influence très nuisible sur la structure et l'évolution pédologique du sol forestier: c'est en grande partie aux coupes de taillis de chênes des " rapailles " qu'on peut attribuer l'acidification et la podzolisation, sur les versants exposés au sud des Basses-Vosges [3]. De même, LACHAUSSÉE a montré que les coupes de taillis simple, sur sol compact, étaient suivies d'un effet très défavorable sur la structure, et qu'elles provoquaient le tassement du sol et la remontée du niveau du Gley. [6].

$2^{\circ}$ Utilisation d'essences acidifiantes, produisant un humus défavorable. - Il s'agit surtout de résineux, notamment du Pin sylvestre et de l'Epicéa, lorsque leur action n'est pas corrigée par un feuillu introduit en mélange: les exemples de dégradation provoquée par les peuplements purs de résineux, en climat atlantique, sur roche-mère siliceuse filtrante, sont maintenant trop connus pour qu'il soit nécessaire d'insister. Rappelons la progression très rapide de la podzolisation sur certaines argiles à silex, sur les sables de Sologne et des Landes, sous l'influence de la monoculture des pins. En ce qui concerne l'Epicéa, cette essence provoque très fréquemment une dégradation de la structure, notamment lorsqu'elle est employée en peuplements purs sur roche-mère acide et imperméable, en climat humide et froid: ainsi, dans le pays de Bade, en forêt de Villingen, sur grès bigarné compact (alt. $780 \mathrm{~m}$ ), nous avons pu remarquer que les bouquets d'Epicéa sont caractérisés de manière constante par un sol tourbeux à Gley: des taches de Sphagnum coïncident rigoureusement avec les bouquets d'Epicéa. Au contraire, 
sous les peuplements de Sapin, on observe un sol brun bien aéré et à humus peu acide.

Dans le même ordre d'idées, Richard a pu noter, sur les sols morainiques du plateau suisse, une diminution considérable de la porosité - notamment la porosité non capillaire - sous les peuplements purs d'Epicéa, par rapport aux peuplements de Chêne voi$\sin 5[7]$.

\section{Les conséquences de la dégradation de la structure.}

Elles peuvent être de deux ordres, suivant qu'on se trouve en présence d'un sol pauvre ou, au contraire, riche en colloïdes argileux. Dans le premier cas, le lessivage s'accentue et fait rapidement place au phénomène de la podzolisation, provoqué par l'acidification accentuée de l'humus qui se transforme en humus brut ou Mor. Ce phénomène ayant été étudié antérieurement, nous n'y reviendrons pas ici [4]. Dans le second cas, un tassement accentué se produit, à la suite de la destruction des agrégats; la baisse importante de la porosité non capillaire en est un sûr indice : la perméabilité du sol décroît alors considérablement, de sorte qu'il a tendance à se saturer d'ean en période humide; c'est ainsi que se forme un plan d'eau temporaire, proche de la surface, qui subsiste pendant toute la période humide, mais s'abaisse progressivement en saison sèche jusqu'à disparition complète par drainage très lent.

En même temps, le profil se modifie; l'humus devient tourbeux et se disperse: le sol prend une teinte générale grisâtre, dans la zone superficielle. A une faible profondeur, les phénomènes de réduction des sels de fer provoquent la (" marmorisation ) du sol: des traînées grisâtres à orientation générale verticale apparaissent sur un fond jaune-rouille; dans les cas plus graves, l'horizon marmoricé se transforme en Gley: les zones gris verdâtre, plus étendues et plus marquées, tranchent alors nettement sur les taches rouille vif du profil.

La végétation, qui envahit le sol, reflète cette évolution défavorable; elle est, en général, constituée par un tapis de Graminées sociales, qui exercent, à l'égard des jeunes semis, une concurrence pour l'eau extrêmement nocive en période sèche; les Agrostis (Agrostis alba et A. canina) colonisent les premiers ces sols à structure dégradée: ils sont relayés dans les cas graves, par la Molinie (Molinia coerulea).

Un exemple particulièrement démonstratif de dégradation de la structure a pu être observé récemment en forêt de Tronçais, à la suite d'une coupe de régénération, datant d'une quinzaine d'années, dans une zone de quelques ares, où la régénération n'a pas réussi: Nous comparerons le sol et la végétation de cette station, en voie de dégradation (parcelle $20-4^{\circ}$ série), à ceux du vieux peuplement encore non entamé, dans une station située à une centaine de mètres de la précédente (Réserve de Morat - parcelle 6). D'après les anciennes descriptions de parcelles, on peut être assuré que les conditions 
étaient les mêmes dans les deux stations, avant la mise en régénération de la parcellé 20.

Profil et végétation du vieux peuplement (vieille futaie de Chêne de 200 ans - sous-étage de Hêtre).

$\mathrm{A}_{1}$ - épaisseur $5 \mathrm{~cm}$ : humus doux, brun foncé, structure grumeleuse.

$A_{2}$ - épaisseur $50 \mathrm{~cm}$ : brun limoneux, structure en petits agrégats.

$\mathrm{BG}$ - épaisseur $20 \mathrm{~cm}$ : Gley compact, reposant sur un conglomérat siliceux imperméable.

Végétation d'humus doux: Houx - Ronce - Melica uniflora.

Profil et végétation de la trouée.

$\mathrm{A}_{1}$ - épaisseur Io $\mathrm{cm}$ : horizon tassé, sans structure nette, gris foncé, infiltré d'humus en voie de dispersion.

$\mathrm{A}_{2}$ - épaisseur $20 \mathrm{~cm}$ : limoneux, structure particulaire, gris clair.

$\mathrm{G}^{2}$ - Gley très compact.

Végétation: Molinie par tache - Agrostis alba - Carex pilulifera.

La comparaison des deux profils met en évidence une évolution très défavorable de la structure, à la suite de la dénudation du sol. Par rapport au sol brun forestier primitif à Gley profond, le sol de la trouée est devenu un sol lessivé à Gley superficiel, dont les horizons supérieurs sont fortement tassés et de couleur grisâtre.

La constitution du plan d'eau temporaire est d'autant plus nuisible à la vie des arbres, notamment à celle des régénérations, qu'il est plus proche de la surface. En hiver, la zone saturée d'eau limite la profondeur de l'enracinement, car elle provoque l'asphyxie des racines. Par contre, la perméabilité étant diminuée, les pertes d'eau par ascension capillaire et par évaporation sont deux à trois fois plus fortes que dans une station draînée [I]. En été, au contraire, le plan d'eau temporaire s'assèche par drainage progressif ; les phénomènes de remontée capillaire cessent complètement, faute de réserve d'eau libre en profondeur, et les arbres, dont les racines ont dû se localiser dans les couches très superficielles du sol, souffrent de la sécheresse.

Ainsi, les sols compacts, à structure dégradée, sont asphyxiants en hiver et, au contraire, desséchants en été; ils sont très défavorables aux régénérations, d'autant plus qu'ils se couvrent fréquemment d'un tapis de Graminées sociales exerçant une forte concurrence pcur l'eau, à l'égard des semis.

\section{IIJ. - LUTTE CONTRE LA DÉGRADATION DE LA STRUCTURE}

D'après ce que nous avons dit, c'est Phumus qui conditionne l'état de la structure du sol forestier; dans une forêt en bon état, l'humus doux ou l'humus calcique maintiennent une structure favorable. Si l'humus se modifie, par acidification, par défaut d'aération, o11 s'il disparait, la structure se dégrade.

Tous les efforts du forestier doivent donc tendre à maintenir, coûte que coûte, l'humus en bon état. Dans ce but, il dispose de deux 
sortes de moyens: $I^{\circ}$ les moyens “préventifs ) à longue échéance, dont les effets s'accumulent pendant toute la vie du peuplement ; $2^{\circ}$ les moyens curatifs susceptibles d'améliorer rapidement une situation compromise (par exemple en présence d'une couche épaisse d'humus brut): ces moyens sont suivis d'effets immédiats favorables et ils permettent, dans un court laps de temps, d'assurer les régénérations. Mais, à longue échéance, ils agissent défavorablement sur la structure, si la reconstitution du peuplement, en l'espèce la régénération, n'est pas assurée de façon immédiate; ces moyens doivent donc être mis en cuvre avec précaution.

\section{Moyens préventifs à action progressive.}

Nous n'insisterons pas sur la solution classique, suffisamment connue, de réalisations de peuplements mélangés résineux-feuillus, dont l'humus est touj ours beaucoup plus favorable à l'activité biologique que celui des peuplements résineux purs. Nous nous bornerons à dire un mot d'une solution nouvelle, qui pourrait être essayée dans certaines circonstances; elle vise à considérer le peuplement artificiel de résineux comme une véritable culture. Celle-ci consiste à travailler fréquemment le sol, à lui incorporer engrais et amendements, entre les lignes de résineux, qui doivent être suffisamment espacées; de telles opérations ont pour effet de ralentir les processsus de lessivage, d'éliminer la végétation défavorable (Ericacées) et de lui substituer une végétation nouvelle, améliorante de l'humus et régénératrice de la structure (Légumineuses). Dans certains cas, on peut même provoquer l'extension sur la surface du sol forestier d'une véritable prairie, constituée d'espèces alibiles, si celles-ci peuvent être maintenues par une humidité suffisante et un pâturage régulier.

En Allemagne, on pratique ainsi une vraie " culture » de l'Epicéa. En France, dans les Landes de Gascogne, des essais sont actuellement en cours, sous légide de la Direction des services agricoles de la Gironde, travaillant en liaison avec la Station de Recherches de l'Ecole Forestière: il est possible que, dans certaines stations privilégiées (sol constamment frais et sans alios), on arrive à créer de véritables prés-bois de Pin maritime.

Rappelons, à ce propos, que ies prés-bois de montagne sont caractérisés par un sol de type brun à humus doux, parfaitement aéré, qui offre des propriétés incomparablement supérieures aux sols de forêts denses à sous-bois d'Ericacées (citons, par exemple, dans les Pyrénées-Orientales, la forêt de Pin sylvestre de la Matte des Angles, ainsi que la plupart des Mélézeins alpins). La méthode dont nous parlons, tend à rapprocher les peuplements artificiels de résineux de cet idéal théorique du pré-bois: elle ne sera, bien entendu, applicable qu'à certains cas particuliers d'humidité suffisante (atmosphère et sol), pour assurer la vie de la pelouse pâturée. 


\section{$2^{\circ}$ Moyens curatifs à effets rapides.}

Ce sont les méthodes classiques qui permettent d'améliorer une situation compromise (forêt dégradée et clairiérée, tourbière ou lande sur sol à humus brut), afin de permettre à la forêt d'occuper le sol à nouveau.

Deux cas sont alors à distinguer, suivant qu'on se trouve en présence d'un sol à humus brut mais bien drainé, ou d'un sol compact, mal aéré et mal drainé.

\section{- Sol à humus brut, en station drainée.}

Les diverses méthodes utilisables sont les suivantes: coupes brutales éclairant le sol dans un peuplement fermé - incinération ménagée et incomplète aboutissant à une combustion partielle de l'humus - travail du sol accompagné ou non d'un chaulage.

Tous ces procédés ont pour effet de provoquer une décompositicn rapide de l'humus, suivie d'un relèvement du $\mathrm{pH}$ et d'une minéralisation intense de l'azote, qui devient assimilable pour les jeunes semis: l'effet immédiat est donc très favorable; mais il est à peine besoin de souligner qu'il est de courte durée; ces pratiques provoquent une disparition plus ou moins complète de l'humus, donc un tassement du sol; les bases et l'azote minéral libérés risquent d'être progressivement lessivés, si le sol est sableux. Enfin, le travail du sol augmente momentanément son aération, mais si le découvert persiste quelques années, un nouveau tassement se produit et la structure devient plus mauvaise que dans les parcelles non travaillées [2]. Aussi, les procédés curatifs doivent-ils être mis en nuvre avec précaution. Ils ne sont efficaces que si la forêt réoccupe à nouveau très rapidement le sol - qu'il s'agisse de repentolement artificiel ou de régénération - faute de, quoi au bout de quelques années, le remède s'avère pire que le mal.

\section{- Sol compact, en station mal drainée.}

Les mesures précédentes doivent alors être complétées par un assainissement $d u$ sol: dans les cas peu graves, un simple labour en billons, permettant l'égouttage du sol par les " dérayures " qu'il crée, est suffisant; dans les cas les plus graves, la création d'un véritable réseau de fossés s'avère indispensable. La reconstitution du peuplement, qui est ainsi rendue possible, permet une amélioration progressive de la situation, en abaissant le plan d'eau temporaire et en fabriquant un humus favorable à la structure. L'intrcduction de résineux résistant à l'asphyxie des racines (Epicéa Thuya géant, Pin Weymouth et, dans le Sud-Ouest, Pin maritime) doit être complétée par la création de bouquets intercalés ou d'un 
sous-étage de feuillus, également adaptés à la station: Chêne rouge d'Amérique, Aune glutineux, Tilleul à petites feuilles, Bouleau pubescent.

\section{CONCLUSION}

Dans la vie de tout peuplement forestier, il est possible de distinguer deux phases:

I $^{\circ}$ Une phase de longue durée, de vieillissement du peuplement au cours duquel un humus doux de type Mull s'accumule sur le sol; c'est une phase de reconstitution progressive de la structure, celleci devant apparaître d'autant meilleure que le peuplement est plus vieux, si la sylviculture est bien conduite.

$2^{\circ}$ Une phase de courte durée, de régénération du peuplement au cours de laquelle, à la suite de la minéralisation rapide de l'humus forestier, la structure a tendance à se dégrader: si les propriétés physiques du sol sont défavorables, une évolution régressive peut alors s'amorcer conduisant, soit à une acidification et à un lessivage ( sol siliceux filtrant), soit à une ( marmorisation ) et à la formation d'un Gley (sol compact).

Il est alors essentiel que le sol forestier reste dénudé et exposé au solcil le moins de temps possible, et le moins souvent possihle; il importe de rét1ssir les coupes de régénération très rapidement, de façon à orienter, dans les délais les plus brefs, l'évolution du sol forestier vers une nouvelle phase constructive de la structure.

Ph. Duchaufour.

\section{BIBLIOGRAPHIE}

I. Baver. - Soil physics. $2^{\mathrm{e}}$ édit., John Wiley and sons inc. N. York, 1948, $400 \mathrm{p}$.

2. H. BÜrGER. - Bodenverbesserungsversuche. Mitt. der Schweiiz. Anst. f. d. forstliche Versuchswesen, t. 28, 1952, p. II-87.

3. $\mathrm{Ph}$. Duchaufour. - La dégradation des sols sur les versants chauds dans les Basses-Vosges. Rev. For. Franç., février 1951, p. I03-109.

4. Ph. Duchaufour. - Lessivage et podzolisation. Rev. For. Franç., octobre I95I, p. 647-652.

5. $\mathrm{Ph}$. Duchaufour. - De l'influence de la chaleur et des radiations sur l'activation de l'humus forestier. Rev. For. Franç., 1953, p. 204-212.

6. E. Lachaussée. - Les sols sous la dominance de l'eau et la forêt. Rev. For. Franc., mai 1950, p. 246-273.

7. F. Richard. - Physikalische Bodeneigenschaften natürlich gelagerter Rissmoränewaldböden unter verschiedener Bestockung. Journal forestiẹ suisse, avril-mai 1953, p. I54-172. 